FORMATION Formation emploi

Revue française de sciences sociales

140 | octobre-décembre 2017

L'éducation à l'esprit d'entreprendre en questions

\title{
Formation à l'entrepreneuriat dans le secondaire français : quelles réalités politiques, du supranational au local?
}

Entrepreneurship education in secondary education, in France: political realities from supranational level to local level

Ausbildung zum unternehmerischen Denken und Handeln in weiterführenden

Schulen in Frankreich: die politischen Realitäten - überstaatlich bis lokal

Formación en emprendedorismo en el secundario francés: ¿qué realidades

políticas de lo supranacional a lo local?

\section{Sylvain Starck}

\section{OpenEdition}

Journals

Édition électronique

URL : http://journals.openedition.org/formationemploi/5240

DOI : 10.4000/formationemploi.5240

ISSN : 2107-0946

Éditeur

La Documentation française

Édition imprimée

Date de publication : 31 décembre 2017

Pagination : 127-145

ISSN : 0759-6340

Référence électronique

Sylvain Starck, «Formation à l'entrepreneuriat dans le secondaire français : quelles réalités politiques, du supranational au local ? », Formation emploi [En ligne], 140 | octobre-décembre 2017, mis en ligne le 31 décembre 2017, consulté le 21 décembre 2020. URL : http://journals.openedition.org/ formationemploi/5240 ; DOI : https://doi.org/10.4000/formationemploi.5240 


\section{Formation à l'entrepreneuriat dans le secondaire français : quelles réalités politiques, du supranational au local ?}

SYLVAIN STARCK Maître de conférences en sciences de l'éducation, Laboratoire Interuniversitaire des sciences de l'Éducation et de la communication (LISEC), équipe ATIP (Travail, Activité, Identité

Professionnelle)

Résumé

Formation à l'entrepreneuriat dans le secondaire français : quelles réalités politiques, du supranational au local ?

Suite aux assises de l'entrepreneuriat, en écho à des préconisations européennes, François Hollande (2013) annonce la mise en place d'un programme sur l'entrepreneuriat dans le secondaire. Qu'en est-il suite à la réforme du collège de 2016 ? Nous rappelons le contexte dans lequel un tel programme est appelé à se développer, en France, en interrogeant sa rhétorique. Puis, nous caractérisons le développement d'une formation à l'entrepreneuriat dans le secondaire, en France, du point de vue des politiques éducatives nationales et régionales. Enfin, nous interrogeons sa mise en œuvre dans la région Hauts-de-France.

Mots clés : politique de l'éducation, contenu de formation, création d'entreprise, enseignement secondaire, région, approche locale, politique régionale, savoir professionnel

Abstract

Entrepreneurship education in secondary education, in France : political realities from supranational level to local level

Following the "Conference on Entrepreneurship», echoing European recommendations, François Hollande (2013) announces the establishment of an entrepreneurship education program in secondary education. What about further the lower secondary school reform of 2016? We recall the context in which such a program is called to develop, in France, by questioning his rhetoric. Then, we characterize the development of training in entrepreneurship in the French secondary education, from the point of view of national and regional educational policies. Finally, we wonder about its implementation in the Hautsde-France region.

Keywords: education policy, training content, business start-up, secondary education, region, local approach, regional policy, professional knowledge

Economic Journal of Literature: I 21 ; I 28 ; L 26

Traduction : Auteur. 
Dans son discours du 29 avril 2013, en clôture des assises de l'entrepreneuriat, le président François Hollande précisait le rôle que l'école devait tenir dans le développement de l'entrepreneuriat, en France : "Notre premier devoir, c'est donc de stimuler l'esprit d'entreprise, l'initiative, dans tous les domaines. C'est d'abord le rôle de l'école. (...) Il est donc prévu, de la sixième à la terminale, un programme sur l'entreprenariat ». Cette nouvelle mission confiée à l'école est à resituer dans une histoire qui a fait de l'école française non seulement le creuset de la Nation, mais aussi un vecteur essentiel de sa puissance économique. Or, l'entrepreneuriat se présente aujourd'hui comme l'activité qui permet de tirer la meilleure plus-value d'une « idée » et donc de soutenir avec succès le défi d'une telle économie. Afin de "mobiliser tous les talents", selon les propos de F. Hollande dans ce même discours, les formations à l'entrepreneuriat sont appelées à dépasser leur périmètre actuel - écoles de commerces et d'ingénieurs principalement - pour se diffuser dans l'ensemble des formations initiales. Comme l'indique le communiqué de presse ministériel du 29 avril 2013, ces assises "attestent une mobilisation sans précédent en faveur des entreprises, facteur essentiel de croissance et d'emploi ». Implicitement, c'est donc l'essor d'un esprit d'entreprise, soit « le développement de compétences propres à susciter le projet de devenir entrepreneur" (ChampyRemoussenard, 2012) - selon une acception restreinte de l'entrepreneuriat ${ }^{1}$ - qui prime du point de vue politique. Une formation à l'esprit d'entreprendre visant le développement d'un état d'esprit orienté vers une création de valeur non exclusivement économique (selon une acception large de l'entrepreneuriat) se justifie par sa capacité à soutenir, à terme, l'esprit d'entreprise.

Or, trois ans après la tenue de ces assises, il est possible d'interroger les répercussions éventuelles d'une telle prise de position dans l'enseignement secondaire français. Comment une éducation à l'entrepreneuriat (EE), considérée sous ces deux acceptions, est-elle prise en compte aujourd'hui dans les enseignements du secondaire, et notamment au collège, suite à la réforme de 2016 ? Celle-ci, outre un réaménagement des temps d'enseignement disciplinaire et de l'accompagnement personnalisé, institue la mise en place d'enseignements pratiques interdisciplinaires (EPI), de la cinquième à la troisième ${ }^{2}$. Précisons d'emblée que nous analysons ici le développement de telles formations en dehors de filières spécifiques d'enseignement afin de comprendre leur éventuelle généralisation dans le second degré d'enseignement, après les prises de position de F. Hollande en 2013. Les nouveaux programmes d'enseignement structurés par le socle commun de connaissances, de compétences et de culture, défini en 2015 et soutenant la réforme du collège de 2016, manifestent-ils une orientation de l'école vers une telle formation ? Ce texte vise à caractériser l'éducation à l'entrepreneuriat dans le paysage français, au prisme des politiques éducatives qui y sont associées. Il s'agit finalement de rendre en partie intelligibles les rela-

1. Voir aussi Pepin (2011b).

2. L'arrivée de Jean-Michel Blanquer au ministère de l'Education nationale, en mai 2017, marque toutefois une inflexion notable quant à la mise en œuvre de cette réforme : assouplissement des obligations en matière d'EPI et rétablissement des classes bilangues notamment. 
tions qui s'établissent entre différents niveaux d'action, allant du supranational à la mise en ouvre locale d'un dispositif de formation.

Pour ce faire, dans un premier temps, nous exposons les positions politiques supranationale et nationale en termes d'EE. À cette étape de notre réflexion, il s'agit d'identifier et de soumettre à la critique la structure argumentative qui justifie ce nouveau champ de pratique éducative. Dans un deuxième temps, nous tentons de caractériser le développement d'une EE dans le second degré d'enseignement, en France, du point de vue de la politique éducative mise en œuvre au niveau national. Dans un troisième temps, nous nous intéressons au développement d'une EE dans la région Hauts-de-France, celle-ci faisant en effet l'objet d'une politique territoriale particulièrement soutenue. Nous interrogeons ici la singularité de ce cas qui ne peut prétendre à être représentatif des autres réalités régionales. Pour finir, nous examinons la mise en ouvre d'une formation à l'entrepreneuriat dans cette même région. À cet effet, nous mobilisons une recherche menée en 2012-2014, dans l'académie de Lille.

Pour pallier la difficulté des changements d'échelle opérés tout au long de l'analyse, nous considérons la manière dont chaque niveau prend en compte trois questionnements essentiels liés à ce champ de pratique émergent, et qui sont partagés invariablement par tous les acteurs : comment susciter l'adhésion des autres acteurs et rendre cette formation souhaitable ? Comment opérationnaliser ce champ de pratique éducatif ? Comment diffuser les pratiques mises en œuvre? Il sera ainsi possible de confronter les logiques mises en œuvre à ces différents niveaux et faire apparaître, en partie, leurs relations.

\section{Une rhétorique transnationale déclinée dans le contexte français}

Après avoir mis en évidence les grandes lignes argumentatives structurant les discours transnationaux en faveur de l'EE, nous analysons leur déclinaison dans le contexte national français.

\subsection{Un circuit argumentatif en faveur d'une éducation à l'entrepreneuriat}

Pour comprendre le développement d'une EE dans le secondaire, en France, il est nécessaire de mettre au jour et d'interroger les éléments de discours qui le justifient. Or, la diffusion de cette culture entrepreneuriale fait l'objet d'un processus mondialisé (Champy-Remoussenard, 2012), "les motifs de développement de l'éducation à l'entrepreneuriat [étant] relatifs à des enjeux sociaux, comportementaux, éducatifs et d'orientation qui semblent faire consensus dans la classe politique aux niveaux transnationaux, nationaux et locaux" (Champy-Remoussenard, 2015, p. 23). Il convient donc de se replacer dans le contexte élargi des discours nationaux et supranationaux. 
La lecture de différents textes qui font référence met en évidence un circuit argumentatif qui justifie, au plus haut niveau, l'essor de telles formations.

Le plan d'action "Entrepreneuriat 2020 », porté par la Commission européenne, affirme que "pour renouer avec la croissance et retrouver un niveau d'emploi élevé, l'Europe a besoin d'un plus grand nombre d'entrepreneurs" (Commission européenne, 2013), d'autant plus que "depuis 2008, l'Europe subit les conséquences de la plus grave crise économique des cinquante dernières années". Cette argumentation est reprise par un rapport de l'OCDE sur l'entrepreneuriat (OCDE, 2013). En effet, " avoir de l'activité économique suppose aussi d'avoir le développement des entreprises. Car c'est une évidencé, mais parfois mieux vaut rappeler ce qui va de soi: ce sont les entreprises qui créent la richesse, qui créent l'activité et qui créent donc l'emploi» (F. Hollande, 2013). Pour ce faire, le plan d'action « Entrepreneuriat 2020 » identifie alors un premier levier qui vise à " promouvoir l'éducation et la formation à l'entrepreneuriat afin de soutenir la croissance et la création d'entreprises». Il s'agit de développer l'esprit d'initiative et d'entreprise, l'une des huit compétences clés du socle européen pour l'éducation et la formation tout au long de la vie ${ }^{4}$, qui témoigne de la capacité des individus à "transformer des idées créatives en acte entrepreneurial» (Commission européenne, 2016).

En France, le développement d'une EE se retrouve ainsi associé, depuis 2012, à une politique volontariste pour l'innovation, inscrite dans le cadre du pacte pour la compétitivité, la croissance et l'emploi.

À ce stade, il convient de souligner le fort recouvrement des éléments rhétoriques adoptés, tant au niveau national que supranational, pour susciter l'adhésion des acteurs sociaux à des visées clairement néolibérales (Tanguy, 2016). Or, ces éléments rhétoriques doivent ici être considérés comme des discours mobilisateurs plus soucieux de

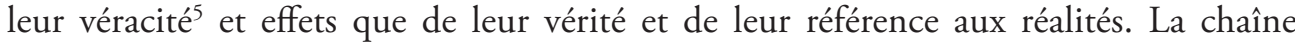
argumentative semble implacable dans sa logique, chaque nouvel argument reposant sur l'évidence du précédent. Or, cette chaîne peut rapidement être mise en défaut par une analyse critique, la rendant ainsi caduque dans son ensemble.

Selon le rapport sur l'entrepreneuriat de l'OCDE en 2013, "renouer avec la croissance et retrouver un niveau d'emploi élevé " demande qu'un plus grand nombre d'individus expriment leur esprit d'entreprise, véritable fer de lance d'une puissance économique retrouvée, tant au niveau de la zone euro qu'au niveau national, si l'on se réfere au début du circuit argumentatif. Un tel raisonnement considère, de manière indistincte, que toute transformation d'une idée créative en acte entrepreneurial permet de renouer avec

3. Souligné par nous.

4. Le Parlement européen et le Conseil de l'Union européenne émettent, le 18 décembre 2006, des recommandations sur les compétences clés pour l'éducation et la formation tout au long de la vie, en définissant notamment un ensemble de huit compétences de base.

5. Dans le sens d'apparaître comme socialement acceptables et donc d'être communément tenus pour vrais. 
la croissance. Or, la crise de 2008 n'est-elle pas imputable, en large partie, à une activité entrepreneuriale - ici financière, liée à l'idée "créative " de la titrisation des crédits immobiliers et à son extension - dont les effets se sont révélés désastreux sur les marchés de l'emploi ou sur les défaillances des entreprises, après 2008 notamment ${ }^{6}$ ? À moins de se conformer à la théorie économique de la " création destructrice » (Schumpeter, 1935), il s'agit à tout le moins de considérer que toute transformation d'une idée créative en acte entrepreneurial n'est pas de nature à participer à l'essor économique d'une population ou à une création de valeur profitable à tous.

Ce qui nous conduit à interroger une seconde évidence inscrite dans le circuit argumentatif : "Ce sont les entreprises qui créent la richesse, qui créent l'activité et qui créent donc l'emploi». Cet énoncé est pourtant loin d'aller de soi, opérant des réductions trompeuses.

La première réduction est celle selon laquelle les richesses sont créées par l'entreprise. Tout ce qui a de la valeur dans une société est-il du ressort de biens et de services produits par des entreprises ? À moins de réduire toute organisation sociale au modèle de l'entreprise, une réponse affirmative n'est pas envisageable. Question d'autant plus vive que l'école, souvent publique, interpellée ici, devrait, selon ce schéma argumentatif, tendre vers une forme entrepreneuriale si elle veut participer à une production de richesse.

La seconde réduction consiste à dire que ce sont les entreprises qui créent l'emploi. Là encore, le propos procède d'une réduction liée à l'échelle d'analyse. Si les entreprises créent, in fine, l'emploi, c'est en raison d'un travail institutionnel qui offre les conditions favorables à leur développement (Sen, 1993).

Le propos, en énonçant des " évidences ", entérine le fait que l'école participe à la production de richesse à condition de participer au développement entrepreneurial, négligeant ainsi les autres formes de valeurs mises en jeu dans l'espace éducatif. Il laisse dans l'ombre la construction des conditions institutionnelles favorables à une économie entrepreneuriale, travail de construction auquel participe l'école. Quelle légitimité accorder alors aux acteurs de l'éducation dans une EE ? Quelle place ce travail et ces savoirs occupent-ils en regard d'une culture entrepreneuriale ? Quels contours donner à cette culture ? Une EE ne peut ainsi éviter de prendre en charge des questions politiques essentielles - et ce, à tous les niveaux d'action - au risque de s'apparenter à un processus d'acculturation sans réelle distance critique.

6. Ainsi, selon Fougère et $a l$. (2013), toutes cohortes confondues, la crise est responsable, en France, de $27 \%$ des défaillances d'entreprises dans le commerce de détail, $35 \%$ dans les transports, $43 \%$ dans l'industrie et $46 \%$ dans la construction. Elle a particulièrement impacté les jeunes entreprises participant à l'innovation et à la création d'emploi. 
Après avoir resitué les orientations et les effets de croyance portés aux plus hauts niveaux politiques, il s'agit de comprendre leur déclinaison dans les autres niveaux d'action, et notamment d'opérationnalisation.

\subsection{Déclinaison nationale d'une formation à l'entrepreneuriat dans le secondaire français}

Le rapport européen de 2016, "Formation à l'entrepreneuriat à l'école en Europe " (Commission européenne, 2016), constitue un analyseur pertinent des visées opératoires déployées à l'échelon supranational. Au-delà d'actions locales, d'initiatives non coordonnées et touchant un public restreint, les incitations au niveau européen visent à diffuser l'EE de manière massive et coordonnée à l'ensemble des écoliers, collégiens, lycéens, mais aussi des enseignants dans le cadre de leurs formations. Ce changement d'échelle est censé permettre aux individus d'être mieux armés face à un marché de l'emploi en crise, en les invitant à exprimer leurs talents, et aux États d'engager un retour vers la compétitivité et la croissance.

Les différentes parties du rapport tracent, en creux, les premiers contours opérationnels d'une EE pleinement intégrée dans les cursus de formation : acquis d'apprentissage, méthodes d'enseignement et d'apprentissage, financements, curriculum, etc. Le rapport expose une $\mathrm{EE}$ au sens large et restreint : trois catégories d'acquis sont déclinées sous forme d'attitudes (confiance en soi, sens de l'initiative), de compétences (planification, créativité, etc.) et de connaissances (évaluation des opportunités par exemple) entrepreneuriales. Les méthodes associées obligent à des apprentissages actifs impérativement éloignés des «méthodes pédagogiques traditionnelles ». Qu’en est-il finalement de l'opérationnalisation d'une EE, en France, au niveau des prescriptions?

Sur le territoire national, la politique éducative en la matière est marquée par une dichotomie entre enseignement secondaire et enseignement supérieur. La formation dans le supérieur est bien mieux structurée avec la mise en place, notamment, d'un "référentiel de compétences sur l'entrepreneuriat et l'esprit d'entreprendre " (ministère de l'Enseignement supérieur et de la Recherche, 2011) ou du statut d'étudiant-entrepreneur qui, dans le cadre des pôles étudiants pour l'innovation, le transfert et l'entrepreneuriat (PEPITE), permet à tout étudiant de développer son projet entrepreneurial intégré à sa formation, tout en bénéficiant d'un accompagnement spécifique. Dans le secondaire, l'EE se trouve prise dans des formes plus obliques, les euphémismes "esprit d'entreprendre » ou " capacité à entreprendre " marquant l'usage officiel (Champy-Remoussenard, 2012). La tonalité des prescriptions reste inchangée en 2016. Dans le nouveau socle de connaissances, de compétences et de culture, l'EE se trouve essentiellement prise en charge dans le domaine 3 , associé à la formation de la personne et du citoyen. Sous la rubrique "Responsabilité, sens de l'engagement et de l'initiative ", il est indiqué que "l'élève sait prendre des initiatives, entreprendre et mettre en ceuvre des projets, après avoir évalué les conséquences de son action; il prépare ainsi son orientation future et sa vie d'adulte ». Parmi 
les quatre parcours créés lors de la réforme du collège, c'est au niveau du " parcours avenir ", associant le projet d'orientation et la découverte du monde économique et professionnel, que nous trouvons des éléments relatifs à une EE sur l'ensemble du second degré. En France, celle-ci ne fait donc toujours pas l'objet d'un programme d'enseignement spécifique ${ }^{7}$ et obligatoire, en dépit de la prise de position publique du Président de la République et des conclusions des assises de l'entrepreneuriat.

$\mathrm{Du}$ point de vue des acquis, les textes considèrent avant tout une EE au sens large (esprit d'initiative visant toute création de valeur). En cela, l'opérationnalisation visée au niveau national se distingue nettement de celle envisagée au niveau supranational. Comme le précise l'arrêté du $1^{\text {er }}$ juillet 2015, le parcours avenir « se fonde sur l'acquisition de compétences et de connaissances relatives au monde économique, social et professionnel, dans le cadre des enseignements disciplinaires et des formes spécifiques d'enseignements diversifiés" dont " l'acquisition de connaissances et des compétences à entreprendre, au sens notamment de découvrir, choisir, créer, agir et mettre en auvre ". Il s'agit de "développer son sens de l'engagement et de l'initiative " dans une démarche de projet. Les prescriptions visent ainsi une EE au sens large. Elles s'inscrivent en effet dans une logique de projet déjà largement présente dans l'espace éducatif et qui peut conjoncturellement, à l'initiative des acteurs de terrain, prendre pour objet la création d'entreprise ou d'une activité économique et répondre à une formation à l'esprit d'entreprise, comme y autorisent certains textes (le parcours avenir ou la circulaire encadrant la mise en ouvre du projet pluridisciplinaire à caractère professionnel - PPCP - en lycée professionnel font explicitement référence au dispositif des mini-entreprises). Quant aux dispositifs de formation à l'entrepreneuriat des enseignants, ils restent pour l'heure très limités. Au niveau national, le Centre d'études et de recherches sur les partenariats avec les entreprises et les professions (CERPEP) dispense quelques formations. Au niveau du M@gistère, ${ }^{9}$ les actions sont en cours d'élaboration.

Comment finalement expliquer cet écart entre une volonté politique affichée en 2013 et une politique éducative, en 2015 et 2016, qui accorde une place relativement marginale à une formation à l'esprit d'entreprise dans le secondaire ? La question se pose d'autant plus que l'enseignement supérieur bénéficie aujourd'hui d'un soutien et d'une organisation institutionnels explicites en direction de l'entrepreneuriat étudiant. Trois éléments explicatifs ${ }^{10}$ sont proposés.

7. Pour l'UE, seules la Lituanie et la Roumanie sont dans ce cas (Commission européenne, 2016).

8. Souligné par nous.

9. M@gistère organise, depuis 2013, une partie de l'offre de formation continue des personnels enseignants du premier et second degré.

10. Bien que ces éléments nous semblent tout à fait recevables, il ne nous est pas possible d'affirmer s'ils signalent les facteurs explicatifs les plus significatifs. Seule une analyse menée auprès des acteurs engagés au niveau national, dans ces politiques éducatives, permettrait de le préciser. 
Les écarts entre une volonté portée au plus haut niveau de l'État, dans la lignée des incitations supranationales, et la politique effective en matière d'EE, révèlent des jeux d'acteurs, des compromis, des résistances, des glissements de sens opérés au niveau des décideurs centraux. Les écarts constatés signalent une activité de médiations multiples (Fenet-Buisson \& Pons, 2012), opérées au niveau central par les différents acteurs concernés (Ministre, Inspection générale de l'Éducation nationale - IGEN -, cabinet ministériel, directions du ministère de l'Éducation nationale, etc.). Si ces médiations ne sont accessibles dans notre démarche que par inférence, l'enquête de terrain évoquée ciaprès, réalisée dans une académie, permet toutefois de spécifier la forme et la nature des médiations opérées aux niveaux académique et local.

D'un point de vue culturel, le milieu enseignant veille à son indépendance vis-à-vis des exigences du monde professionnel et des pressions que celui-ci peut exercer sur les finalités et les contenus d'enseignement. Les instances syndicales sont traditionnellement sur ces mêmes positions et ne peuvent qu'être pour le moins réservées vis-à-vis de telles préconisations ${ }^{11}$. En cela, une formation explicite et programmée à l'esprit d'entreprise susciterait actuellement de vives critiques d'une partie du monde enseignant. En termes stratégiques, il est possible de lire le développement d'une formation à l'esprit d'entreprise laissée à l'initiative des enseignants ou d'équipes pédagogiques, voire d'établissements, comme une manière de progressivement sensibiliser le monde enseignant par les pairs, et plus largement le corps social. À terme, il s'agit de susciter l'adhésion selon une logique horizontale, la logique verticale de type «top-down» étant jugée, pour l'heure, politiquement trop risquée.

D'un point de vue conjoncturel, le nouveau socle de connaissances, de compétences et de culture, ainsi que les parcours éducatifs mettent l'accent sur les questions morales et de citoyenneté. La capacité à entreprendre est ainsi placée sous l'égide de la formation de la personne et du citoyen. Entreprendre s'inscrit dans une perspective morale conséquentialiste : l'élève apprend à entreprendre "après avoir évalué les conséquences de son action ». Cet accent mis sur la formation morale et à la citoyenneté - directement en écho à la lutte engagée aujourd'hui contre le terrorisme - réactive une finalité historique de l'école, en France, et pointe ici une dépendance au sentier (Palier, 2014). L'EE pose des questions politiques fondamentales. En effet, les modèles de socialisation associés à une société entrepreneuriale se distinguent nettement des processus de socialisation prévalant dans l'école française, historiquement rattachés à une perspective durkheimienne de l'éducation. Ainsi, les savoirs en jeu visent moins, en France, l'empowerment et l'apprentissage par l'expérience que le raisonnement droit et une formation morale de l'esprit et du caractère (Meuret, 2007, pp. 42-46). Engager une EE interpelle ainsi

11. Un enseignant rencontré au cours de notre enquête signale ainsi avoir publié un article sur l'entrepreneuriat dans une revue syndicale enseignante, tout en restant quelque peu étonné que cela ait pu se faire. 
l'histoire de l'école, et donc les métiers, les pratiques, les cultures qui la constituent, incitant à des jeux d'acteurs.

En laissant l'initiative aux acteurs de terrain, les politiques nationales semblent finalement faire le pari d'une résolution possible des tensions évoquées ci-dessus au niveau local. D’où le choix de déléguer l'opérationnalisation des orientations politiques directement aux acteurs du terrain (front-office). On peut ici parler de politique territorialisée dans le sens où des politiques élaborées au niveau central mobilisent directement les territoires dans leur opérationnalisation (Autès, 1995).

\section{Une analyse territoriale du développement de l'éducation à l'entrepreneuriat dans le secondaire}

Pour clore la contextualisation de la mise en œuvre locale d'une EE, il nous faut encore y adjoindre l'échelon régional, initiateur lui aussi d'une politique territoriale ${ }^{12}$ en matière d'EE. Les analyses qui suivent s'appuient sur une enquête de terrain portant sur la mise en ouvre d'un dispositif phare de l'EE, à savoir les mini-entreprises $\left(\mathrm{ME}^{13}\right)$.

\subsection{Des politiques territoriales en faveur d'une EE}

Pour rendre compte de ce niveau d'action, nous étudions le cas de la région Nord-Pasde-Calais, intégrée depuis 2016 à la région Hauts-de-France. Elle est la seule région de France à avoir obtenu, pour 2013, le label " Région Européenne Entreprenante ", créé en partenariat avec la Commission européenne et avec le soutien de parties prenantes actives au niveau de l'UE (European Association of Craft, Small and Medium-sized Enterprises, etc.) afin de favoriser le développement de l'entrepreneuriat en région ${ }^{14}$.

La région Nord-Pas-de-Calais a en effet mis en place, dès 2006, une stratégie en faveur de l'entrepreneuriat sur son territoire. Elle poursuit aujourd'hui cette orientation politique et économique. L'objectif affiché, en 2013, est de "construire une vision collective et un socle commun favorisant le développement de l'initiative économique " (Baeza et al., 2016, p. 111). Assurer le renouveau du tissu économique d'une région industriellement sinistrée requiert notamment, selon cette stratégie, de "sensibiliser la population régionale à la création, à la reprise et à la transmission d'entreprises", l'enjeu étant "de favoriser le passage à une culture de l'initiative et le développement de l'esprit d'entreprise ${ }^{15}$ ».

12. Une politique territoriale est construite depuis, à partir et vers des territoires, ce qui la distingue des politiques territorialisées toujours subordonnées aux pouvoirs centraux.

13. Pour plus de précisions sur ce type de dispositif, voir par exemple Pepin (2011b).

14. Signalons que cette politique de labellisation permet de relier niveau supranational et régional en omettant le niveau national.

15. Schéma régional de développement économique, adopté par le Conseil Régional le 24 novembre 2005. 


\section{Encadré 1 : Une enquête de terrain menée dans l'Académie de Lille}

L'enquête de terrain, de nature exploratoire $(*)$, a été menée entre septembre 2012 et mars 2014, dans l'académie de Lille, en partenariat avec le Rectorat de Lille et dans le cadre de financements du Fonds social européen (FSE). La recherche a mobilisé plusieurs démarches : une observation à visée ethnographique d'une mini-entreprise sur l'année de son fonctionnement (soit une douzaine d'observations accompagnées de deux entretiens avec l'enseignant référent et deux entretiens avec deux groupes de trois et quatre élèves), treize entretiens semi-directifs auprès de différents acteurs engagés dans le développement des mini-entreprises - ME (pilotes académiques ou membres d'une association partenaire, chargés de mission, enseignants), deux entretiens de groupe réalisés auprès de lycéens. Les entretiens abordaient plusieurs dimensions : éléments biographiques, motifs d'engagement dans l'EE, mise en mots de pratiques associées à I'EE, relations aux autres acteurs et effets perçus de l'EE. Un questionnaire en ligne a été adressé à plus d'une centaine d'enseignants, soit la quasi-totalité des référents des ME pour la session 2012-2013. On compte 68 répondants pour 110 enseignants sollicités. Ce questionnaire visait, notamment, à caractériser la population concernée et à identifier les représentations associées à I'EE. Rappelons que dans le cadre d'une recherche de type exploratoire, les données sont analysées ici selon une perspective tendancielle; il s'agit en effet avant tout de comprendre certaines configurations d'action, sans présager de leur éventuelle représentativité.

${ }^{*}$ ) : Les formations à l'entrepreneuriat constituent, en France, à la date de l'enquête, un champ quasi inexploré du point de vue des sciences de l'éducation. Contrairement aux travaux sur les " éducations à ", qui sont en plein essor depuis plusieurs années, l'EE reste peu explorée par les chercheurs en sciences de l'éducation. Il existe a contrario de nombreux travaux dans d'autres espaces nationaux (Québec, États-Unis, Royaume-Uni, etc.). En France, les travaux existants sont issus d'autres disciplines (sciences de gestion notamment) (Champy-Remoussenard, 2012).

Cette volonté de développer l'esprit d'entreprise en région se traduit alors par l'élaboration et la mise en œuvre du projet "Innovons et Développons l'Esprit d'Entreprendre " (IDEE), porté par l'Académie de Lille. L'objectif est de développer, chez les élèves, les "compétences de l'entrepreneuriat». Cet affichage clair d'une formation aux compétences dites entrepreneuriales et orientée, in fine, vers la puissance économique du territoire, tranche avec les tournures plus euphémisées relevées au niveau national.

En cela, la politique territoriale relative à l'EE se distingue nettement des orientations nationales par un affichage plus clair d'une visée économique et d'une formation à l'esprit d'entreprise. La distinction s'opère aussi par une opérationnalisation bien plus soutenue en la matière : mobilisation de nombreux acteurs et constitution de réseaux fonctionnels (c'est par exemple trois enseignants, chargés de mission auprès de la cellule école-entreprise, qui ont vocation à promouvoir l'EE en région Hauts-de-France), élaboration et mise en œuvre d'actions de formation à destination des enseignants, mise en place et labellisation d'actions pédagogiques spécifiques. Ainsi, si la politique nationale fait la part belle à l'initiative des acteurs de terrain, sans réel accompagnement en la matière, la politique territoriale (portée par le Conseil régional et le Rectorat), 
bien plus structurée, devient prépondérante et figure, dans sa singularité, comme une expérimentation en matière $\mathrm{d}^{\prime} \mathrm{EE}^{16}$.

Dans la suite de nos analyses, nous tentons de rendre compte de la manière dont les différents acteurs proches du terrain (pilotes et enseignants) déclinent, dans leurs pratiques, cet entrelacement de politiques, territorialisée et territoriale.

\subsection{Entre l'échelon politique et les enseignants : des médiations réalisées par les acteurs}

Pour comprendre comment les enseignants s'emparent de l'EE, nous analysons le travail des différents acteurs qui opèrent des médiations entre l'espace des politiques éducatives portées au niveau régional - prenant en compte les politiques, nationale et territoriale - et l'espace des mises en œuvre dans les classes. Il s'agit de comprendre quelle forme adopte finalement la politique éducative liée à l'EE lorsqu'elle parvient au niveau des enseignants, en considérant les trois catégories d'analyses que sont la mobilisation, l'opérationnalisation et la diffusion. Nous nous inspirons ici des travaux de BuissonFenet et Pons (2012) sur les médiations professionnelles dans l'analyse des politiques éducatives.

Trois acteurs - que nous désignons comme des pilotes de l'EE en région, et que nous avons interviewés dans notre enquête - jouent un rôle important en la matière ; deux sont rattachés au Rectorat. Le premier travaille au sein de la Délégation académique aux enseignements techniques (DAET), le deuxième exerce ses activités en détachement du monde de l'entreprise, le troisième enfin est directeur d'une association partenaire ${ }^{17}$.

S’agissant de la mobilisation des différents acteurs, les deux premiers pilotes disent opérer un travail "d'explication de texte " auprès de leurs différents interlocuteurs. Lors des diverses réunions auxquelles il a pris part, en lien avec l'EE, le premier pilote déclare en avoir mesuré "les freins " et les "leviers ". La "stratégie " qu'il a contribué à élaborer face aux différentes instances syndicales, aux chefs d'établissements, aux corps d'inspection, est de proscrire le recours au mot " entreprise ", pour systématiquement parler d'esprit d'entreprendre : "S'entreprendre pour soi et ensuite pour les autres avec son intégration possible et future dans le monde économique ", rendant cette issue accessoire et conjoncturelle. Ceci permet alors de faire "sauter des verrous", et notamment d'avoir une écoute bienveillante de milieux syndicaux «durs». Cette euphémisation du lexique - en écho à la stratégie nationale - se poursuit lorsque le second pilote précise que l'EE revient, dans le fond, à sensibiliser, à " donner envie ", laissant l’interlocuteur compléter implicite-

16. Précisons toutefois qu'il ne s'agit pas de faire de ce cas un modèle en matière d'EE. Il s'agit avant tout de comprendre les logiques mises en œuvre par les acteurs de terrain dans leurs contextes professionnels. La place des partenaires extérieurs est par exemple variable selon les régions.

17. Dans le souci de préserver l'anonymat de ces personnes, les statuts ne seront pas plus précisés ici. 
ment le propos. Le flou entretenu autorise un adressage "positif» à une grande diversité de partenaires. L'action du premier pilote est soutenue par trois enseignants/chargés de mission qui reprennent ces éléments de langage visant deux objectifs complémentaires : démobiliser les résistances potentielles, susciter des vocations partout sur le territoire. Ces trois médiateurs, dont le statut bivalent indique le rôle clé qu'ils jouent dans le dispositif, ont pour objectif de rassurer les chefs d'établissement et les enseignants qu'ils rencontrent lors de réunions de bassin, de convaincre certains d'entre eux de l'intérêt d'une EE. Les chefs d'établissement constituent de même un maillon essentiel. Ils autorisent en effet la mise en lien des chargés de mission avec les enseignants et incitent parfois ces derniers à engager une action d'EE en raison de l'image favorable qu'ils peuvent en retirer dans un espace de quasi-marché ${ }^{18}$ (Barrère, 2006). Une fois le terrain ouvert, les chargés de mission passent le relais aux accompagnateurs et animateurs des parties prenantes partenaires et porteuses de l'action. Les médiations s'opèrent alors autour des dispositifs - dénommés " outils» par les pilotes - et que nous concevons comme des instruments (Rabardel, 2005) (voir partie suivante).

La situation du troisième pilote se distingue de celle du premier interlocuteur en raison de sa forte dépendance aux acteurs du monde entrepreneurial et de la région, qui financent en grande partie ses actions, mais aussi de sa dépendance non moins forte aux instances académiques qui maîtrisent le marché sur lequel l'association partie prenante «place ses produits ». Cette situation est comparable pour toutes les associations partenaires, de manière plus ou moins aiguë en fonction de la force des dépendances. Les acteurs de la région et du monde entrepreneurial expriment clairement le souhait de développer, chez les jeunes, une culture de l'entreprise, des comportements entrepreneuriaux, afin de susciter des carrières de futurs porteurs d'activités économiques. Ce sont bien ces objectifs qui justifient les importants investissements réalisés ${ }^{19}$. A contrario de ses homologues dans d'autres régions, dans ce contexte singulier, il dit intégrer, dans son activité, cette dynamique de création d'entreprise et la mettre en avant dans les supports de communication. Les parties prenantes d'une EE, du fait de leur extériorité institutionnelle à l'Education nationale et du soutien politique territorial en matière d'entrepreneuriat, peuvent ici porter des discours moins ambigus sur une formation à l'esprit d'entreprise. C'est aussi le cas des acteurs du monde de l'entreprise qui vont intervenir lors de différentes actions.

Apparaissent donc des situations multiples où les discours - en fonction des situations et des interlocuteurs - varient entre la mise en avant d'une EE, mettant principalement l'accent sur l'esprit d'entreprendre, quand à d'autres moment, et dans d'autres arènes, les

18. Les établissements scolaires s'inscrivent aujourd'hui, en fonction des réalités locales, dans des mécanismes de concurrence, tout en restant sous la dépendance de financements publics et de coûts de formation non négociables (Dupriez et Dumay, 2011).

19. Pour l'action phare des ME, le coût de l'une d'entre elles avoisine les $3500 €$, intégrant $900 €$ liés à la tenue du salon régional. La charge financière supportée par les établissements est de $300 €$ annuels, le reste étant pris en charge par des financements du monde entrepreneurial et de la Région. 
discours sont franchement orientés vers une formation à l'esprit d'entreprise. Sur le terrain local, il n'existe donc pas un discours unique qui encadre l'EE, mais une multiplicité de discours développés de manière stratégique. Les instances de pilotage qui regroupent, selon des configurations variées, acteurs des établissements, représentants du Rectorat, du Conseil général et du monde de l'entreprise, engagent un travail incessant de reconfiguration du lexique, selon des modalités d'essai-erreur. Les pilotes régionaux restent ainsi attentifs aux évolutions du champ de pratique et de ce qui devient socialement acceptable et désirable. C'est selon cette perspective qu'il faut entendre les propos précédents du premier pilote. Il explique, par exemple, que "l'idée de ces réunions [avec les différents partenaires et instances représentatives] était vraiment de mesurer les freins et les leviers au développement de l'entreprenariat, bien comprendre comment il fallait agir pour quion puisse se faire comprendre et expliquer la dynamique. Histoire de ne pas partir et se heurter à un mur. Ça a été le plus gros travail et je pense quion a été relativement efficace ". Les profils variés présents dans le pilotage opérationnel (enseignants, administratifs, responsables d'associations partenaires, professionnel de l'entreprise en détachement, membres du Mouvement des entreprises de France - MEDEF) permettent d'assurer ce travail rhétorique et idéologique multi-niveaux.

Les médiations, que l'on devine à partir des interviews réalisées, laissent apparaître non pas le déroulement d'un discours uniforme descendant, mais un champ de pratiques, partagé et en construction, qui cherche pour l'heure son équilibre. Ainsi, si L. Tanguy (2016) met en évidence, et à juste titre, la prégnance de discours massifs et descendants rattachés à une idéologie néolibérale, elle laisse en grande partie dans l'ombre le poids des singularités opérant aux différents niveaux d'action. ${ }^{20}$

Les dernières médiations que nous évoquerons dans cette partie sont celles réalisées par les enseignants eux-mêmes. Le questionnaire (cf. encadré 1) permet de préciser leur profil : le sexe, l'âge, l'ancienneté ne constituent pas des facteurs discriminants. Les professeurs certifiés sont surreprésentés - $57 \%$ des répondants - pour $4 \%$ d'agrégés et $17 \%$ de professeurs de lycée professionnel. Du point de vue des disciplines, $20 \%$ des enseignants appartiennent à la filière éco-gestion, plus de $30 \%$ enseignent en sciences de la vie et de la terre (SVT), en technologie et physique, les autres disciplines ou les enseignements techniques étant plus faiblement représentés.

$\mathrm{Au}$ cours du déroulement de la $\mathrm{ME}$, les enseignants sont amenés à communiquer en direction de leurs collègues, soit lors de réunions institutionnelles (Guy, certifié de l'enseignement technique, 15 ans d'ancienneté et Amélie, contractuelle depuis 11 ans, tous deux en lycée professionnel, ou encore Marc, professeur des écoles en IME, 20 ans d'ancien-

20. Selon nous, l'approche de l'auteure mobilise implicitement une théorie de transferts institutionnels (Buisson-Fenet, Pons, 2012) qui suppose le déplacement, d'un système politique à l'autre, des connaissances et des instruments attachés à l'action publique et masque en partie le poids des autres acteurs concernés. 
neté), ou bien à la suite du salon régional récompensant les meilleurs projets (Paul, certifié en français, collège privé) ou encore lors des activités de la ME, vente notamment, (Catherine, certifiée en mathématiques, collège, 9 ans d'ancienneté). Aux dires des personnes interviewées, ces médiations - qui sont en partie accessibles dans les entretiens suscitent parfois l'adhésion de quelques collègues dans l'établissement. La charge de travail que représente une $\mathrm{ME}$ oblige d'ailleurs les enseignants volontaires à recruter certains de leurs collègues. Il semble toutefois, comme l'indique par exemple Véronique (professeure de lycée professionnel - PLP - en gestion, 30 ans d'ancienneté), que l'originalité et la lourdeur du projet limitent les vocations et par là-même une diffusion de l'EE dans les établissements.

De même, des médiations importantes sont réalisées par les enseignants lors du salon régional récompensant les meilleures $\mathrm{ME}$ de l'Académie. Cette manifestation, dont le coût avoisine les $100000 €$, se déroule dans les locaux imposants de Lille Grand Palais et réunit plusieurs centaines de personnes. Elle est tout autant le point d'orgue du dispositif valorisant l'implication des différents acteurs, une opération de communication auprès des partenaires existants et futurs qu'une fidélisation des participants. Les pilotes du dispositif profitent de la tenue du concours régional pour y inviter des acteurs de l'enseignement et étendre le vivier des enseignants et établissements impliqués dans l'EE. Certains des enseignants que nous avons interviewés se remémorent leur première venue au salon : l'ambiance festive, l'effervescence des élèves, la satisfaction des enseignants lors des échanges impromptus et les signes de reconnaissance auxquels ceux-ci ont droit (les organisateurs les invitent, par exemple, à monter sur scène pour y être ovationnés).

\subsection{Des médiations réalisées par les instruments}

Rendre compte du déploiement de l'EE requiert toutefois d'interroger le rôle que jouent les instruments qui y sont liés et les médiations multiples qu'ils opèrent (Rabardel, 2005). Nous pensons plus particulièrement à l'un d'entre eux, emblématique de l'EE : le dispositif des ME, entendu ici comme "construit socio-technique" " (Albéro, 2010), élaboré et affiné au cours de ses mises en œuvre successives par l'opérateur « Entreprendre pour apprendre " (EPA), en association avec les différents partenaires. En effet, pour Albéro (2010) «le dispositif se présente d'emblée comme l'artefact fonctionnel qui matérialise une organisation particulière d'objets, d'acteurs, de structures et de systèmes de relations, en fonction des objectifs de formation dans une situation donnée ". Cette place stratégique des instruments dans le développement d'une EE est pleinement intégrée par le deuxième pilote qui, en adoptant une perspective exclusivement pragmatique au cours de l'entretien, foca-

21. La perspective sociotechnique permet de prendre en compte les phénomènes de construction interdépendante de sujets et d'objets intégrant dimensions instrumentales, cognitives et sociales. Un dispositif de formation reconduit ainsi, dans une sorte de philosophie obligée, des «significations socialement valorisées de rationalisation et d'organisation fonctionnelle qui assurent son succès » et dans le même temps, des "effets implicites de normalisation et de standardisation des conduites individuelles" (Albero, 2010, p. 57). 
lise son propos sur la question des " outils ". En polarisant la réflexion sur les dimensions fonctionnelles et opératoires de l'EE, elle élude ainsi des questions politiques potentiellement clivantes. La ME, en tant qu'instrument, réalise de manière économique plusieurs choses remarquables :

- Elle opérationnalise l'EE selon deux processus. D’une part, elle conduit l'activité des acteurs impliqués. Dans la première année de fonctionnement, la mini-entreprise constitue un dispositif fonctionnel qui impose son rythme aux enseignants, situation qui n'est d'ailleurs pas complétement dépassée lors de la deuxième année, au vu des observations réalisées : "Alors, c’est à nous de nous adapter à cet encadrement qui a déjà été prévu par EPA, mais de l'adapter ici. Parce qu'on est parti, on ne savait rien. Du moins, on ne savait pas comment ils voyaient les choses, donc on découvre tout doucement (...)» (Guy et Amélie). C'est la "dimension productive » de l'instrument qui opère une médiation entre le sujet et le but à atteindre (Rabardel, 2005). D'autre part, l'usage de la $\mathrm{ME}$ comme instrument participe à la formation des enseignants. C'est la dimension constructive de celui-ci qui médiatise le rapport du sujet à lui-même, via son rapport au dispositif ;

- Le dispositif et sa labellisation par le Rectorat évitent les aspects polémiques de deux manières. Ils déplacent le cœur de la réflexion du " pourquoi faire » vers le « comment faire ". Ils garantissent la conformité du dispositif au cadre règlementaire et limitent les dimensions potentiellement polémiques de l'action ;

- La ME participe à l'objectif de diffusion de l'EE par une certaine standardisation de sa mise en ouvre. Adoptant la logique d'une formation par projet, sous cet aspect, elle est mobilisatrice car en partie familière.

Dans les faits, la politique territoriale portée au niveau du Rectorat consiste à privilégier la constitution et la labellisation d'un panel d'actions en direction de tous les niveaux et filières d'enseignement, systématiquement avec des opérateurs extérieurs liés au monde économique. Cette stratégie répond aux trois objectifs de mobilisation, d'opérationnalisation et de diffusion de l'EE. Si cette standardisation d'outils semble opérer une uniformisation des pratiques en matière d'EE, l'analyse plus fine de leur usage met en évidence le poids relatif de ses protagonistes - notamment élèves et enseignants - dans une politique en actes de l'EE.

En effet, l'instrument a la faculté d'opérer des médiations opératoires entre les différents acteurs (Rabardel, 2005 ; Rabardel, Samurçay, 2006), tout en laissant suffisamment de liberté à ces derniers pour déterminer en pratique ses usages réels, permettant à chaque configuration d'acteurs d'opérer un positionnement singulier. Il s'agit ainsi de distinguer l'artefact - ensemble de contraintes qui s'imposent au sujet, guide et objet d'appropriation dans l'action - de l'instrument généré par les mises en ouvre locales où "l'instrument réel du sujet résulte d’une élaboration progressive" (Rabardel, Samurçay, 2006, p. 43). 
Cette dimension est particulièrement visible dans les observations réalisées, croisées avec les propos de Fabien (certifié en sciences physiques, 11 ans d'ancienneté), en charge de la ME observée. Bien qu'il minimise son rôle dans les choix opérés lors de la construction de la ME, il s'est, dans les faits, largement emparé de la ME pour inciter les élèves à engager une activité entrepreneuriale ancrée dans une perspective citoyenne. Sa position vient en écho du « Parcours avenir " qui place l'EE sous l'égide d'une éducation à la citoyenneté. Les produits confectionnés et vendus par la ME sont ainsi porteurs de messages prônant le respect des différences. Or, suite au salon régional au cours duquel aucun prix n’a été décerné aux élèves, Fabien repense son usage de la $\mathrm{ME}$ : «Est-ce que ça fait vraiment entreprise qui veut faire des profits? Là, tout à coup, on peut penser que non, on vend ça pour une ouvre caritative. Je pense que la personne [du jury] a ressenti cela lorsqu'elle a posé ses questions, de se dire 'est-ce que vous avez vraiment voulu faire une entreprise'?".

Cette réflexion met en évidence les intrications fonctionnelles et axiologiques - et donc politiques - susceptibles d'être activées dans l'usage d'un instrument éminemment plastique. Comme le signale Rabardel (2005, p. 258), "linstrument se charge, pour chaque sujet, d'une multiplicité de sens constitués par l'ensemble des valeurs fonctionnelles et subjectives qui se sédimentent en lui au cours de sa génèse et de ses inscriptions dans l'activité des sujets, valeurs qui se confrontent et s'articulent à celles qui sont héritées de l'histoire sociale des artéfacts et des schèmes ». Prendre en charge une ME revient donc à se glisser dans les structures cognitives qui ont modelé ce dispositif tout au long de son élaboration, à en incorporer et en actualiser les logiques. Dans les plis de l'usage de la ME se logent les rapports que le sujet entretient avec lui-même, la mission à accomplir, les autres, le contexte professionnel, l'histoire du métier. Si les médiations opérées au niveau régional et la politique nationale ont pesé sur les actions réalisables, réalisées et empêchées dans un établissement scolaire, il convient d'intégrer à notre compréhension les choix qui président à la déclinaison pratique des dispositifs.

\section{Conclusion}

Nous avons fait ici le choix de présenter quelques résultats qui visent à expliquer selon quelles tendances l'éducation à l'entrepreneuriat (EE) se développe aujourd'hui, en France, en relevant le défi d'intégrer, dans notre vision de l'EE, l'ensemble des niveaux d'action, du suprational au local.

En écart d'une représentation de l'action publique selon le modèle top-down ou d'une théorie de transferts institutionnels, nous proposons de lire les politiques éducatives liées à l'EE à l'aune d'un processus global de médiations complexes qui rend compte d'un objet lui-même complexe. Il convient ainsi de comprendre l'articulation des politiques supranationale, nationale (territorialisée), territoriale et locale.

Des analyses qui précèdent, il ressort que la réalisation d'une EE est une compétence réellement partagée entre les différents acteurs qui réopèrent, chacun à leur niveau, des 
médiations sur des réalités elles-mêmes (re)médiatisées par d'autres acteurs, constituant une réalité éducative complexe. Seule une vision inattentive aux détails peut imaginer une éducation à l'entrepreneuriat ou plutôt ici, une EE double, selon une acception large ou étroite. Il est bien plus juste de parler d'une pluralité d'EE, lorsque l'analyse se montre attentive aux configurations singulières mises en œuvre sur les terrains.

L'analyse du développement de l'EE en région Hauts-de-France, qui fait ici office de cas singulier, permet toutefois d'identifier une caractéristique commune aux médiations réalisées, à savoir une dépolitisation des questions relatives à l'EE. C'est en ce sens que l’on peut lire une présentation de l'EE comme réponse logique, voire évidente - et donc indiscutable - au contexte de crise économique et de concurrence généralisée. L'accord quasi général - et donc difficilement discutable là aussi - autour d'un tel développement de l'EE ${ }^{22}$, participe du même mouvement. Le travail d'euphémisation des textes officiels ou des différents médiateurs territoriaux suit cette même direction de dépolitisation de l'objet. Or, le fait que l'éducation soit par nature au cœur de questions politiques majeures et que cet objet éducatif se trouve largement soutenu par des parties prenantes liées au monde des grandes entreprises (Tanguy, 2016, pp. 178-182 ; Laval \& al., 2012, pp. 175-179) induit a contrario, pour le monde de la recherche, la nécessité de (re)placer les réflexions sur l'EE au cœur d'espaces politisés. Les analyses précédentes exposent en effet une pluralité de niveaux d'action. Chacun d'entre eux constitue le lieu privilégié où s'actualisent potentiellement des questions politiques se distinguant selon leur point de vue : contextes macro-socio-économiques, modèles politiques d'éducation, gouvernance locale, configuration éducative. En cela, une analyse politique de l'EE se doit de prendre en compte l'ensemble de ces niveaux, au risque de privilégier, voire d'imposer, certaines catégories, au détriment d'une lecture globale des transformations et des débats en cours ou empêchés.

\section{Bibliographie}

Albéro B. (2010), "La formation en tant que dispositif : du terme au concept ", in Charlier B., Henri F. (dirs.), La technologie de l'éducation : recherches, pratiques et perspectives, Paris, Presses Universitaires de France, coll. «Apprendre », pp. 47-59.

Autès M. (1995), «Le sens du territoire », Recherches et prévisions, n 39, pp. 57-70.

Baeza C., Champy-Remoussenard P., Dervaux C., Didier C., Gaujard C., Lepers, P. \& Starck S. (2016), "Grand angle sur les pré-incubateurs étudiants : les Hubhouses ", in Phillipart P. (coord.), Ecosystème entrepreneurial et logiques d'accompagnement. Cormelles le Royal, EMS, pp. 108-133.

22. Souligné notamment par le récent rapport de la Commission européenne (2016) sur l'entrepreneuriat à l'école. 
Barrère A. (2006), Sociologie des chefs d'établissement. Les managers de la République, Paris, Presses Universitaires de France, coll. «Education et société ».

Buisson-Fenet H., Pons X (2012), "L'européanisation de l'École française en débat : le cas contrasté de l'évaluation des établissements scolaires ", Politix, n 98, pp. 129-146.

Champy-Remoussenard P. (2015), «Les transformations des relations entre travail, éducation et formation dans l'organisation sociale contemporaine : questions posées par trois dispositifs analyseurs ", Revue française de pédagogie, 190, pp. 15-28.

Champy-Remoussenard P. (2012), "L'éducation à l'entreprenariat : enjeux, statut, perspectives ", Spirale, 50, pp. 39-51

Commission européenne (2016), Formation à l'entrepreneuriat à l'école en Europe. Rapport Eurydice, Bruxelles, Commission européenne.

Commission européenne (2013), Plan d'action " entrepreneuriat 2020 ». Raviver l'esprit d'entreprise en Europe, Bruxelles.

Dupriez V., Dumay X. (2011), "Les quasi-marchés scolaires : au bénéfice de qui ? », Revue française de pédagogie, 176, pp. 83-100.

Fougère D., Golfier C., Horny G., Kremp E. (2013), "Quel a été l'impact de la crise de 2008 sur la défaillance des entreprise ? ", Economie et Statistique, n 462-463, p. 69-97.

Hollande F. (2013), Clôture des assises de l'entrepreneuriat, Discours du Président de la République, Paris, 29 avril.

Laval C., Vergne F., Clémet P. \& Dreux G. (2012), La nouvelle école capitaliste, Paris, La Découverte.

Meuret D. (2007), Gouverner l'école : une comparaison France/États-Unis, Paris, Presses Universitaires de France.

Ministère de l'Enseignement supérieur et de la Recherche (2011), Référentiel de compétences sur l'entrepreneuriat et l'esprit d'entreprendre, Paris.

OCDE (2013), Panorama de l'entrepreneuriat 2013, Éditions OCDE.

Palier B. (2014) ( $4^{\mathrm{e}}$ éd.), "Path dependence (dépendance au chemin emprunté)», in Boussaguet L. et al., Dictionnaire des politiques publiques, Presses de Sciences Po (PFNS), "Références », pp. 411-419.

Pepin M. (2011a), "L'entrepreneuriat en milieu scolaire, de quoi s'agit-il ? ", McGill Journal of Education / Revue des sciences de l'éducation de McGill, vol. 46, n² 2, pp. 303-326.

Pepin M. (2011b), «L'éducation entrepreneuriale au primaire et au secondaire : gros plan sur la micro-entreprise scolaire ", Revue canadienne de l'éducation, n³ 34, pp. 280-300. 
Rabardel P. (2005), "Instruments, activité et développement du pouvoir d'agir », in Lorino P. et al., Entre connaissance et organisation: l'activité collective, Paris, La Découverte, coll. « Recherches », pp. 251-265.

Rabardel P., Samurçay R. (2006), « De l'apprentissage par les artéfacts à l'apprentissage médiatisé par les instruments ", in Barbier J.-M. et al., Sujets, activités, environnements, Paris, Presses Universitaires de France.

Schumpeter J.-A. (1935), Théorie de l'évolution économique, Paris, Dalloz.

Sen A. (1993), "Codes moraux et réussite économique ", Actes de la recherche en sciences sociales, $\mathrm{n}^{\circ} 100$, pp. 58-65.

Tanguy L. (2016), Enseigner l'esprit d'entreprise à l'école. Paris, La Dispute. 